\title{
Large-area traveling-wave photonic mixers for increased continuous terahertz power
}

\author{
E. A. Michael, ${ }^{\text {a) }}$ B. Vowinkel, and R. Schieder \\ Physics Institute, University of Cologne, Zülpicher Strasse 77, 50937 Köln, Germany \\ M. Mikulics, M. Marso, and P. Kordoš \\ Institute of Thin Films and Interfaces (ISG-1), Research Center Jülich, 52425 Jülich, Germany
}

(Received 30 July 2004; accepted 11 February 2005; published online 11 March 2005)

\begin{abstract}
A large-aperture design for terahertz traveling-wave photomixers, continuously pumped free space by two detuned diode lasers, is proposed and experimentally verified for devices based on low-temperature-grown GaAs (LT-GaAs). It combines the advantages of conventional interdigitated small-area structures and traveling-wave devices. An output power of $1 \mu \mathrm{W}$ at the mixing frequency of $1 \mathrm{THz}$ was measured in initial testing, which meets local oscillator power requirements for superconducting heterodyne mixer devices. () 2005 American Institute of Physics.
\end{abstract}

[DOI: $10.1063 / 1.1884262$ ]

Continuous-wave photonic mixer terahertz $(\mathrm{THz})$ radiation sources are basically fast photoconductive switches modulated with the beat frequency of two detuned nearinfrared (IR) diode lasers. ${ }^{1}$ One of the fastest materials to follow the beat frequency in the $\mathrm{THz}$ range is lowtemperature-grown GaAs (LT-GaAs) with photocarrier trap times down to $100 \mathrm{fs}^{2}{ }^{2}$ Besides the corresponding unmatched tuning range, photomixers are also particularly attractive for their all-solid state, noncryogenic, low power consumption, and relative low-cost approach. Therefore, they are interesting as local oscillators (LOs) for heterodyne submillimeter or terahertz receivers ${ }^{3,4}$ based on superconductor-insulatorsuperconductor junctions (SIS) or hot-electron bolometers (HEB). Providing the power necessary for these mixers above $1 \mathrm{THz}$ is a challenge, but is within reach of the current development of LT-GaAs photomixers. The latest report for smallest-area SIS junctions is $p_{\text {pump }} \approx 0.1 \mu \mathrm{W}$ at $1 \mathrm{THz}$ at the mixer ${ }^{5}$ (with a theoretical frequency dependency of $p_{\text {pump }} \sim f^{2}$ ), and for HEBs $p_{\text {pump }} \approx 0.2 \mu \mathrm{W}$ at $1.8 \mathrm{THz}$ at the mixer Si lens ${ }^{6}$ (with an expected frequency dependency of $\left.p_{\text {pump }} \sim f\right)$.

With small-area photonic mixers, consisting of interdigitated metal-semiconductor-metal electrode structures, hereafter MSM [Fig 1(c)], it proved to be difficult to routinely reach these power levels above $1 \mathrm{THz}$. If small-area mixers are used with broadband antennas of load resistance, $R_{a}$, the uncompensated device capacitance, $C$, introduces a rolloff, ${ }^{2,7}$ $\sim 1 /\left[1+\left(2 \pi \cdot R_{a} C \cdot \nu\right)^{2}\right]$, which is usually around $1 \mathrm{THz}$. However, if it is located at the footpoint of resonant antennas, capacitance up to a certain value may be tuned out by the antenna inductance, ${ }^{8}$ and the terahertz power at the resonance frequency, $\nu_{\text {res }}$, follows just the unavoidable rolloff, $\sim 1 /\left[1+\left(2 \pi \cdot \tau_{e} \cdot \nu_{\text {res }}\right)^{2}\right]$, given by the effective response time, $\tau_{e}$, for the electronic current seen locally at the electrodes. This in turn is not identical to the photoelectron trap time, ${ }^{1,9-11}$ but is elongated by the intrinsic transit time of a space-charge dominated current pulse initiated by the shortliving photoelectrons between the electrodes. ${ }^{9,12}$ The restriction on the capacitance first imposes upper limits on the de-

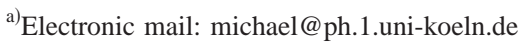

vice area and number of fingers. Because of a destruction intensity of $<1.5 \mathrm{~mW} / \mu \mathrm{m}^{2}$ for LT-GaAs this limits IR pump power to $<100 \mathrm{~mW}$, so that improved passive thermal sinking is essential for small-area mixers, ${ }^{3,13}$ unless cooling to liquid nitrogen temperatures is applied. ${ }^{14}$ Second, it imposes lower limits on the finger spacing which affects conversion efficiency. All together this results in restrictions on the ultimate generated power above $1 \mathrm{THz}$. Power levels up to $2 \mu \mathrm{W}$ at $1 \mathrm{THz}$ have been achieved for tuned structures at a laser power of $90 \mathrm{~mW} .{ }^{8,13}$

To circumvent these IR power and $\mathrm{THz}$ resonance restrictions, it was experimentally demonstrated that a traveling-wave (TW) setup bypasses the device RC constant by establishing a distribution of the mixing process along a coplanar stripline (CPS) through interference fringes propagating phase matched with respect to the $\mathrm{THz}$ wave on the waveguide. ${ }^{15-17}$ The THz output power is then given by

$$
P_{\mathrm{THz}}(v)=\frac{\eta_{r}}{2} \cdot \frac{R_{a} \cdot I_{\mathrm{DC}, e}{ }^{2}}{1+\left(2 \pi \cdot \tau_{e} \cdot v\right)^{2}},
$$

where $\eta_{r}$ is the radiation efficiency of the antenna and $I_{\mathrm{dc}, e}$ is the interference part of the electronic dc photocurrent

$$
I_{\mathrm{dc}, e} \sim \sqrt{P_{\mathrm{IR}, 1} \cdot P_{\mathrm{IR}, 2}} \cdot \tau_{e, \text { trap }}\left(V_{b}\right) \cdot v_{e, \mathrm{dr}}\left(V_{b}\right) / s
$$

which is only a fraction of the total measured photocurrent. Compared to tuned resonant small-area devices with fullwave antenna impedance around $210 \Omega,{ }^{8}$ a factor 3 lower $\mathrm{THz}$ efficiency of broadband TW devices at same photocurrent is given from waveguide impedance around $70 \Omega$. $P_{\mathrm{IR}, 1}$ are the IR powers of the two lasers (usually $P_{\mathrm{IR}, 1}=P_{\mathrm{IR}, 2}$ ), $\tau_{e, \text { trap }}$ is the electron trap time, $v_{e, \mathrm{dr}}$ is the drift velocity and $s$ is the effective photoconductive gap width seen by the electrons in the material (curved field lines). While the trap time increases with voltage (see below), the drift velocity saturates at a field strength of about $2 \mathrm{~V} / \mu \mathrm{m}$, so that $P_{\mathrm{THz}}$ $\sim\left(P_{\text {IR,coupled }} / s\right)^{2}$, making diffraction-limited optics and mode purity a necessity for CPS waveguide mixers. Due to a practical limitation of numerical apertures of diffraction-limited lens systems of $\quad D / f \leqslant 0.6(D=$ diameter of lens, $f$ $=$ focal length), not more than about $65 \%$ of a Gaussian beam can be coupled into the optimal slit width of about $2 \mu \mathrm{m}$. No 


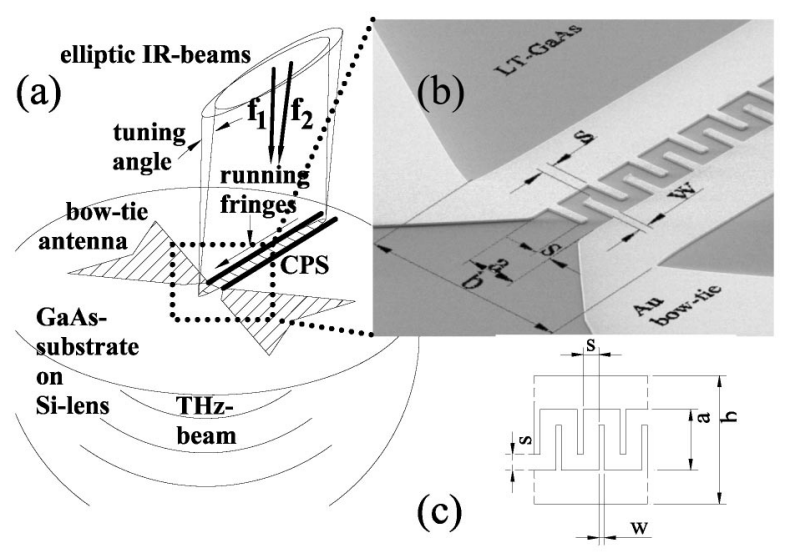

FIG. 1. (a) Velocity-match of IR interference fringes to the THz wave on the CPS is obtained by a tuning-angle between the two heterodyned IR beams with $f_{2}>f_{1}$; (b) scanning electron microscope picture of a TW-MSM structure with inner part of bow-tie antenna; (c) small-area MSM or section of a TW-MSM.

THz efficiency can be gained upon reducing the gap width below that of the Airy spot because then $P_{\mathrm{IR}, \text { coupled }} \sim s$ and $P_{\mathrm{THz}} \approx$ const. Furthermore, the output beam of a commercial semiconductor optical amplifier (SOA) is found to have a higher-mode content of about 50\%, which cannot be coupled into a $2 \mu \mathrm{m}$ gap and decreases THz power from a plain CPS so that it needs to be filtered out, e.g., by introducing a pinhole mode filter after the amplifier.

To bypass these new IR power and THz efficiency limitations, a structure is needed which combines the advantages of a conventional interdigitated small-area structure (wider transversal aperture and smaller gap widths) with those of a waveguide mixer (intrinsic bypass of RC-time-constant and larger area). A solution to this is an interdigitated-finger coplanar stripline (hereafter TW-MSM) depicted in Fig. 1(b), which has a slow-wave behavior and therefore was proposed earlier for velocity-matched longitudinally pumped TW photodetectors. ${ }^{18}$ This attribute, however, is not needed for the vertically illuminated TW mixer structure proposed here, in which velocity matching of the interference fringes to the $\mathrm{THz}$ wave is obtained by adjusting a tuning-angle between the two heterodyned IR beams of different frequency [Fig. 1(a)]. The IR focus line length, biased to short values due to THz absorption, was set to about $150 \mu \mathrm{m}$ FWHM. The astigmatic optics setup ${ }^{19}$ was designed to obtain diffraction limited focusing in the transverse direction.

$e$-beam lithography with a photoresist liftoff technique was used to apply a $10 \mathrm{~nm}-\mathrm{Ti} / 300 \mathrm{~nm}-\mathrm{Au}$ metallization on $1.5 \mu \mathrm{m}$ LT-GaAs grown by molecular beam epitaxy at $250{ }^{\circ} \mathrm{C}$ with $1 \mu \mathrm{m} / \mathrm{h}$ on a $0.3 \mu \mathrm{m} \mathrm{AlAs}$ insulator layer on SI-GaAs and annealed $10 \mathrm{~min}$ at $600{ }^{\circ} \mathrm{C}$. Three different dimensions of the TW-MSM were tested (Fig. 1): $s=1.4,1.6$ and $1.8 \mu \mathrm{m}$ with $w=0.44,0.5$, and $0.56 \mu \mathrm{m}$, respectively. While $a=3 \cdot s, b$ was optimized to match the impedance of a $150-\mu \mathrm{m}$-bowtie antenna $(70 \Omega)$, which was possible after introducing a miter at the transition [Fig. 1(b)]. A commercial time-domain program was used for the simulations.

The THz power spectrum, including higher modes from the bowtie antenna, was measured up to $1500 \mathrm{GHz}$ with a calibrated $4.2 \mathrm{~K} \mathrm{InSb-bolometer} \mathrm{with} \mathrm{a} \mathrm{large-aperture} \mathrm{horn}$ and detector chip, and can be fit to a single-time-constant Lorentz curve (Fig. 2). From this we conclude that the lumped-element RC constant of the structure, expected in the Downloaded 21 Dec 2006 to 134.94.122.39. Redistribution subject

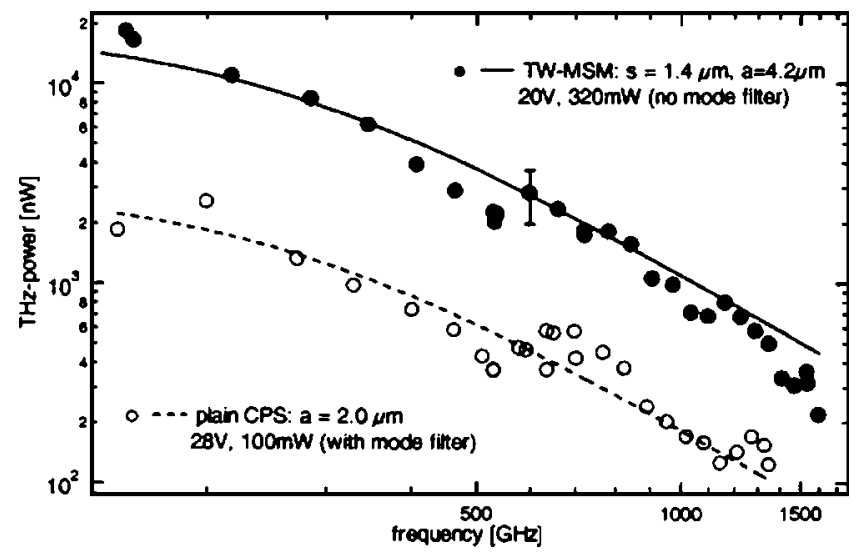

FIG. 2. Calibrated power spectrum of TW-MSM and CPS with bowtie antenna $L=150 \mu \mathrm{m}$. Two bolometer features are noted: a responsivity dip around $500 \mathrm{GHz}$ and the band edge at 1400-1500 GHz. THz power is corrected for calculated bowtie reflection efficiency, but not for reflection losses of the silicon hemispherical lens $(30 \%)$.

order of $100 \mathrm{GHz}$, is completely bypassed, indicating velocity match to the optical interference wave and impedance match to the antenna, so that there is no additional rolloff setting in above $1500 \mathrm{GHz}$ expected, except contributions from unavoidably increasing absorption. However, the $\mathrm{THz}$ losses at about $5 \mathrm{~mm}^{-1}$ were measured to be rather flat between 500 and $1500 \mathrm{GHz}$. The fitted response-time in $P_{\mathrm{THz}}(\nu) \sim 1 /\left[1+\left(2 \pi \cdot \tau_{e} \cdot \nu\right)^{2}\right]$ is $\tau_{e}=0.8 \mathrm{ps}$ at $12 \mathrm{~V} / \mu \mathrm{m}$, while a zero voltage trap time for LT-GaAs grown at $T_{g}$ $=250{ }^{\circ} \mathrm{C}$ and annealed at $600{ }^{\circ} \mathrm{C}$ is expected at $\tau_{e, \text { trap }}$ $=0.66$ ps. $^{11}$

The bolometer was calibrated as follows: The difference of bolometer voltage response to two blackbodies at 300 and at $77 \mathrm{~K}$ was measured and in addition the normalized $\mathrm{THz}$ response spectrum of the bolometer was determined by using it as the detector in a commercial far-infrared Fouriertransform spectrometer. Then the blackbody power within the measured bandpass was calculated at the two temperatures, taking into account the known aperture and solid detection angle of the bolometer horn.

Whereas for a CPS the generated $\mathrm{THz}$ power slightly decreases upon adding the higher modes from the SOA, Fig. 2 shows that a TW-MSM benefits from this additional power to a large extent. However, the increase is lower than expected from $P_{\mathrm{THz}} \sim\left[P_{\mathrm{IR}} \cdot V_{b} /(s+w)\right]^{2}$, because saturation effects are active with respect to bias voltage $V_{b}$ (Fig. 3) and IR power (Fig. 4). As expected, the best $\mathrm{THz}$ power of 1.1 $\mu \mathrm{W}$ at $910 \mathrm{GHz}$ was obtained from the $1.4 \mu \mathrm{m}$ device. Before saturation sets in, the power shows a superquadratic dependence on $V_{b}$ above $4 \mathrm{~V} / \mu \mathrm{m}$, in accordance with the quadratic $I-V$ curve. The $P_{\mathrm{THz}}(V)$ curves above $2 \mathrm{~V} / \mu \mathrm{m}$ fit to

$$
P_{\mathrm{THz}, \mathrm{Fit}}=\frac{\left(c_{0}+c_{1} \cdot V_{b}^{2}\right)^{2}}{1+\left(c_{2}+c_{3} \cdot V_{b}^{2}+c_{4} \cdot V_{b}^{3}\right)^{2}},
$$

assuming a degradation of the photoelectron trap time with electric field to explain voltage saturation above $10 \mathrm{~V} / \mu \mathrm{m},{ }^{19}$ which is supported by our and others' observations of its absence at near-dc frequencies. ${ }^{14}$ Accordingly, the increased voltage saturation of the TW-MSM compared to the CPS is explained by a locally increased electric field at the interdigitated-finger tips and therefore is better described with an additional cubic term. Some differences in the bias AlP license or copyright, see http://apl.aip.org/apl/copyright.jsp 


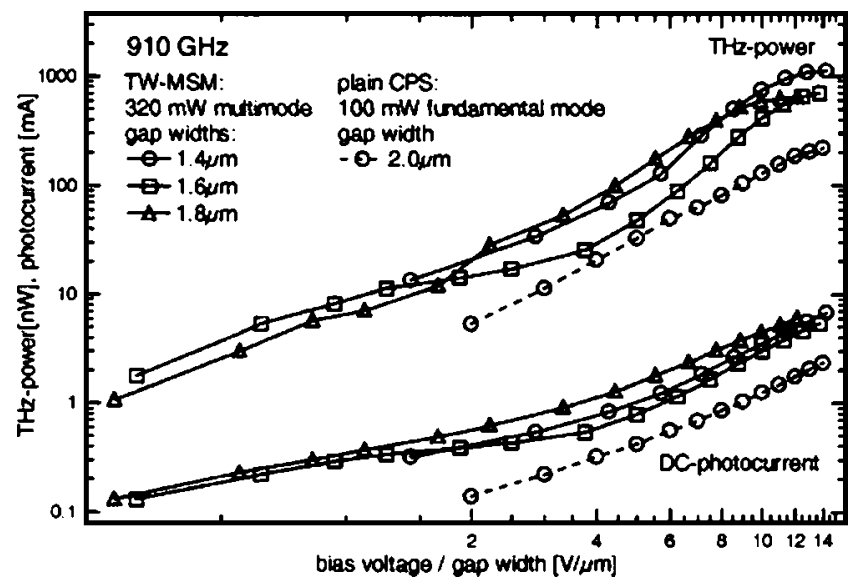

FIG. 3. Comparison of bias behavior between a set of TW-MSM mixers of different gap widths and a CPS mixer.

curves between individual TW-MSM devices (Fig. 3) may be due to variations in the LT-GaAs properties over position on the wafer and contact quality, but increased $\mathrm{THz}$ power towards reduced gap widths is obvious. The $1.6 \mu \mathrm{m}$ device most clearly shows that the photocurrent is governed by three regimes: ${ }^{21}$ (1) an ohmic regime below $1 \mathrm{~V} / \mu \mathrm{m}$; (2) a drift-velocity saturation at $1-4 \mathrm{~V} / \mu \mathrm{m}$; and (3) a quadratic behavior above $E_{q 0}=3 \mathrm{~V} / \mu \mathrm{m}$ due to increase of $\tau_{e \text {,trap, }}$ where $E_{q 0}$ is given by $L \cdot\left(1 / \mu_{e}+1 / \mu_{h}\right) /\left(\tau_{h}-\tau_{e}\right) .{ }^{14}$ Because $\mu_{e} \gg \mu_{h}$ and $\tau_{h} \gg \tau_{e}{ }^{14}$ one may estimate $\mu_{h} \cdot \tau_{h} \approx L / E_{q 0}=5$ $\times 10^{-9} \mathrm{~cm}^{2} / \mathrm{V}$, while $\mu_{e} \cdot \tau_{e} \approx 1.5 \times 10^{-9} \mathrm{~cm}^{2} / \mathrm{V}\left(\tau_{e}=0.66 \mathrm{ps}\right.$, $\mu_{e}=2250 \mathrm{~cm}^{2} / \mathrm{Vs}$ at $T_{g}=250{ }^{\circ} \mathrm{C}$ ), ${ }^{11,22}$ so that about $80 \%$ of the total observed dc photocurrent $I_{\mathrm{dc}}$ is due to holes. This would explain the observed factor of 20 drop of generated power from near-dc (around $3 \mathrm{GHz}$ ) to $150 \mathrm{GHz}$, assuming $\tau_{h} \approx 6$ ps (Ref. 14) in a two-component model for the $\mathrm{THz}$ power $P_{\mathrm{THz}}(\omega) \sim \mid \mu_{e} \cdot \tau_{e} /\left(1+2 \pi i \cdot \tau_{e} \cdot \nu\right)+\mu_{h} \cdot \tau_{h} /(1$ $\left.+2 \pi i \cdot \tau_{h} \cdot \nu\right)\left.\right|^{2}$. The near-dc power, measured through a biastee with a GHz-powermeter with $Z_{\text {load }}=50 \Omega$ is in accordance with the expectation from full modulation of the total measured dc photocurrent $I_{\mathrm{dc}}$, namely $P_{\mathrm{GHz}}$

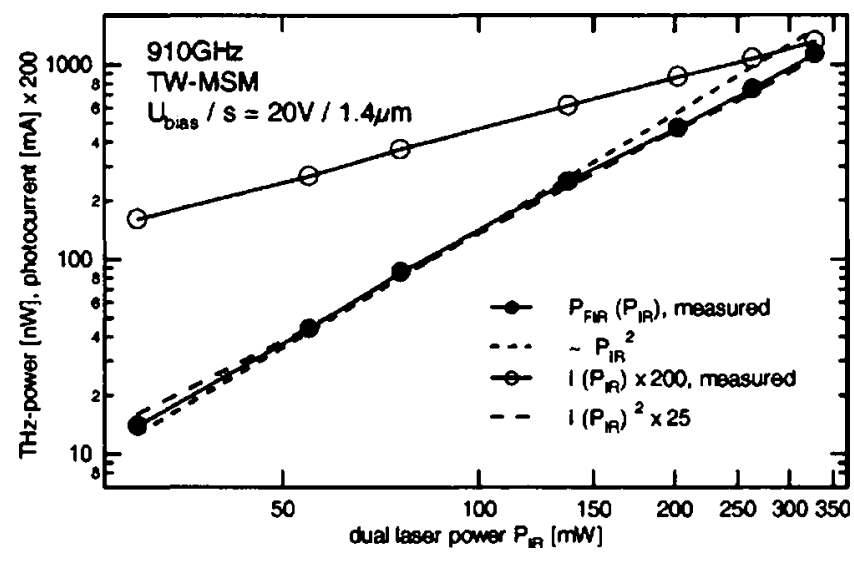

FIG. 4. THz power over combined IR power. The observed deviation from $\sim P_{\mathrm{IR}}{ }^{2}$ at higher IR powers is also present at near-dc frequencies. However, the relation $P_{\mathrm{THz}} \sim I^{2}$ holds up to highest IR powers.
$=1 / 2 \cdot Z_{\mathrm{load}} \cdot\left(I_{\mathrm{dc}} / 2\right)^{2}$, which indicates good interference contrast of the lasers.

We achieved promising results by using an interdigitated-finger CPS structure for improved $\mathrm{THz}$ power from a TW mixer. The expected improvement compared to a CPS within the same LT-GaAs material system is deteriorated by increased voltage saturation due to fringing fields at the fingertips. Because very different maximum bias fields were reported for interdigitated-finger structures, $7,13,14$ improvements on material, layer structure, and contacts are likely to alleviate voltage saturation and to lead to the stateof-the-art $\mathrm{THz}$ efficiencies observed by others previously at lower IR powers. ${ }^{8}$ Therefore, the presented scheme has great potential to largely surpass previously reported power levels beyond $1 \mathrm{THz}$.

The authors acknowledge support from the Deutsche Forschungsgemeinschaft through Grant No. SFB 494 and from the Ministry of Science of the State North Rhein Westfalia, Germany.

${ }^{1}$ K. A. McIntosh, E. R. Brown, K. B. Nichols, O. B. McMahon, W. F. DiNatale, and T. M. Lyszczarz, Appl. Phys. Lett. 67, 3844 (1995).

${ }^{2}$ K. A. McIntosh, K. B. Nichols, S. Verghese, and E. R. Brown, Appl. Phys. Lett. 70, 354 (1997).

${ }^{3}$ S. Verghese, E. K. Duerr, K. A. McIntosh, S. M. Duffy, S. D. Calawa, C.-Y. E. Tong, R. Kimberk, and R. Blundell, IEEE Microw. Guid. Wave Lett. 9, 245 (1999).

${ }^{4}$ M. Wingender, E. A. Michael, B. Vowinkel, and R. Schieder, Opt. Commun. 217, 369 (2003)

${ }^{5}$ A. Karpov, D. Miller, F. Rice, J. Zmuidzinas, J. A. Stern, B. Bumble, and H. G. LeDuc, Fifteenth International Symposium on Space Terahertz Technology, Northampton, MA, USA, April 27-29, 2004

${ }^{6}$ T. M. Klapwijk, R. Barends, J. R. Gao, M. Hajenius, and J J. A. Baselmans, Proc. SPIE 5498, Glasgow, Scottland, 21-25 June 2004.

${ }^{7}$ E. R. Brown, K. A. McIntosh, K. B. Nichols, and C. L. Dennis, Appl. Phys. Lett. 66, 285 (1995).

${ }^{8}$ S. M. Duffy, S. Verghese, K. A. McIntosh, A. Jackson, A. C. Gossard, and S. Matsuura, IEEE Trans. Microwave Theory Tech. 49, 1032 (2001).

${ }^{9}$ I. S. Gregory, C. Baker, W. R. Tribe, M. J. Evans, H. E. Beere, E. H. Linfield, A. G. Davies, and M. Missous, Appl. Phys. Lett. 83, 4199 (2003).

${ }^{10}$ P. Kordoš, A. Förster, M. Marso, and F. Rüders, Electron. Lett. 34, 119 (1998).

${ }^{11}$ H. Němec, A. Pashkin, P. Kužel, M Khazan, S. Schnüll, and I. Wilke, J. Appl. Phys. 90, 1303 (2001).

${ }^{12}$ S. Y. Chou, Y. Liu, and P. B. Fischer, Appl. Phys. Lett. 61, 477 (1992).

${ }^{13}$ A. W. Jackson, Ph.D. thesis, University of California, Santa Barbara, 1999.

${ }^{14}$ E. R. Brown, K. A. McIntosh, F. W. Smith, K. B. Nichols, M. J. Manfra, C. L. Dennis, and J. P. Mattia, Appl. Phys. Lett. 64, 3311 (1994).

${ }^{15}$ S. Matsuura, G. A. Blake, R. A. Wyss, J. C. Pearson, C. Kadow, A. W. Jackson, and A. C. Gossard, Appl. Phys. Lett. 74, 2872 (1999).

${ }^{16}$ S. Matsuura, G. A. Blake, R. A. Wyss, J. C. Pearson, C. Kadow, A. W. Jackson, and A. C. Gossard, Nara, Japan, IEEE Seventh International Conference on Terahertz Electronics Proceedings, Nov. 25-26, p.24 (1999).

${ }^{17}$ J. C. Pearson, P. Chen, and H. M. Pickett, Proc. SPIE 4855, 459 (2003).

${ }^{18}$ E. H. Böttcher and D. Bimberg, Appl. Phys. Lett. 66, 3648 (1995).

${ }^{19}$ E. A. Michael, M. Mikulics, M. Marso, P. Kordoš, H. Lüth, B. Vowinkel, R. Schieder, and J. Stutzki, Proc. SPIE 5498, 525 (2004).

${ }^{20}$ N. Zamdmer, Q. Hu, K. A. McIntosh, and S. Verghese, Appl. Phys. Lett. 75, 2313 (1999).

${ }^{21}$ J. P. Ibbetson and U. K. Mishra, Appl. Phys. Lett. 68, 3781 (1996).

${ }^{22}$ M. C. Beard, G. M. Turner, and C. A. Schmuttenmaer, J. Appl. Phys. 90, 5915 (2001). 